\title{
r \\ Uso de métodos estatísticos e computacionais em pesquisas educacionais utilizando o Moodle: um Mapeamento Sistemático da Literatura
}

\author{
Eduardo Dalcin', Ilse Abegg², Paulo Ceretta ${ }^{2}$ \\ ${ }^{1}$ IFFAR - Instituto Federal Farroupilha - RS - Brasil \\ 2UFSM - Universidade Federal de Santa Maria - RS
}

eduardo.dalcin@iffarroupilha.edu.br,ilse.abegg@ufsm.br, cerettalo@gmail.com

\begin{abstract}
Resumo. Este estudo apresenta os resultados de um mapeamento sistemático da literatura, que buscou investigar o uso de métodos estatísticos e computacionais em pesquisas educacionais utilizando o Moodle, no âmbito nacional e internacional. Após a seleção dos estudos primários, foram analisados 59 trabalhos, os quais registraram as maiores frequências para o uso de medidas descritivas, algoritmo de aprendizado de máquina (Machine Learning) e estudos envolvendo as variáveis: aprendizagem, interação $e$ interatividade. As contribuições mais citadas foram relacionadas com o acompanhamento de estudantes, aprendizado online e inovações tecnológicas nos ambientes virtuais de ensino e aprendizagem. Como limitações registraram-se o aumento do número de variáveis e diferentes análises nos processos de interações. A pesquisa apresentou contribuições relevantes, principalmente para aperfeiçoar e (re)planejar metodologias de ensino a distância em momentos de distanciamento social .

Palavras-chave: Moodle, Algoritmo Computacional, Educação a Distância.
\end{abstract}

\section{Use of statistical and computational methods in educational research using Moodle: a Systematic Mapping of Literature}

\begin{abstract}
This study presents the results of a systematic mapping of the literature, which sought to investigate the use of statistical and computational methods in educational research using Moodle, nationally and internationally. After selecting the primary studies, 59 studies were analyzed, which recorded the highest frequencies for the use of descriptive measures, machine learning algorithm and studies involving the variables: learning, interaction and interactivity. The most cited contributions were: related to the monitoring of students, online learning and technological innovations in virtual teaching and learning environments. As limitations, there was an increase in the number of variables and different analyzes in the interaction processes. The research presented relevant contributions, mainly to improve and (re) plan distance learning methodologies in moments of social distance.
\end{abstract}

Keywords: Moodle, Computational Algorithm, Distance Education.

\section{Introdução}

Segundo Romero, Ventura e Garcia (2008), a quantidade de dados disponíveis no Moodle em estudos a distância vem crescendo, seja a partir do registro de acessos de professores, tutores e estudantes, ou pelo registro de atividades e avaliações realizadas no ambiente. Diante disso, um grande volume de dados é armazenado no banco de dados do Moodle, dificultando análises manuais. Com isso surgem tecnologias para apoiarem e solucionarem essa demanda.

A aprendizagem de máquina (machine learning) é uma das áreas da inteligência artificial que propõe soluções para isso, podendo ser definida como sistemas capazes de adquirir conhecimento a 
partir de dados. Weiss e Kulikowski (1991) definem um sistema de aprendizado de máquina como um algoritmo computacional que toma decisões baseadas na experiência contida em eventos anteriores.

Ademais, o termo aprendizagem de máquina pode ser utilizado por professores e estudantes em diversas atividades, auxiliando o seu desenvolvimento cognitivo. Com a aplicação do machine learning nos recursos do Moodle, também pode-se conseguir analisar o interesse dos estudantes durante o processo de aprendizagem e predizer se ele pretende evadir. Isso é possível com as análises efetivadas pelo sistema em relação à frequência de acessos e tempo de permanência do estudante no ambiente virtual.

Esse método fornece aos professores a oportunidade de reverter a situação, interagindo com o estudante e entendendo o que pode ser realizado para melhorar a sua relação com as atividades ou tratar suas dificuldades.

Diante disso, objetiva-se nesse estudo realizar um mapeamento sistemático sobre o uso de métodos estatísticos e computacionais em pesquisas educacionais utilizando o Moodle como Ambiente Virtual de Ensino e Aprendizagem (AVEA).

Assim, tornam-se relevantes estudos que pretendem analisar o uso de métodos estatísticos e computacionais que envolvem o processo de ensino e aprendizagem em atividades a distância. Fato esse que merece destaque, principalmente para aperfeiçoar estratégias e (re)planejar metodologias de ensino, principalmente em momentos de distanciamento social que vivemos em período de pandemia mundial.

Esse estudo está dividido nos seguintes tópicos: Introdução, explicitando o objetivo da pesquisa e sua importância no contexto educacional a distância. Metodologia de trabalho, descrevendo o processo metodológico do estudo realizado, contendo o processo de busca, string utilizada na pesquisa e condução, após os Resultados e Discussões. E por fim, as Considerações Finais.

\section{Metodologia}

Segundo Kitchenham e Charters (2007), um Mapeamento Sistemático (MS) é uma forma de identificar, analisar e interpretar todas as evidências disponíveis a respeito de uma questão de pesquisa, área, ou fenômeno de interesse. Dentro de um período determinado pelo pesquisador, a fase seguinte é marcada pela busca de fontes. A partir dos resultados obtidos, é necessário definir critérios de inclusão e exclusão com o objetivo de selecionar as fontes de acordo com o propósito de pesquisa. Assim, efetuando-se uma pré-leitura detalhada dos estudos selecionados, extraem-se os dados mais relevantes.

Por fim, chega-se a parte da análise, em que são organizados e depurados os resultados das análises referentes as perguntas de extração. Conforme Kitchenham e Charters (2007), tal análise é capaz de organizar evidências existentes envolvendo um tratamento ou tecnologia. Richter (2019), acrescenta que os cruzamentos são capazes de oferecerem as informações necessárias para resumir o "estado da arte" do objeto de pesquisa, auxiliando o pesquisador na tomada de decisões quanto ao futuro da pesquisa na área. Nos próximos itens apresentaremos as etapas que compõe o MS definido pelos autores, já com os elementos e dados deste estudo.

\subsection{Objetivo e processo de busca}

Esta revisão tem como objetivo identificar quais variáveis utilizadas, contribuições, limitações, métodos, técnicas estatísticas, softwares e algoritmos que são utilizados em pesquisas educacionais envolvendo o Moodle em atividades a distância, no âmbito nacional e internacional. Nesse sentido, o 
trabalho incluiu artigos publicados nos últimos 5 anos (2015 - 2020). Para essa pesquisa, foram selecionadas teses, dissertações e artigos publicados nos anais dos seguintes eventos e periódicos: Congresso Brasileiro de Informática na Educação (CBIE) ${ }^{1}$, Revista Novas Tecnologias na Educação (RENOTE) $)^{2}$, Portal de Periódicos ${ }^{3}$ e Catálogo de Teses e Dissertações da CAPES ${ }^{4}$. Esses portais, eventos e periódicos foram selecionados pela sua grande relevância no contexto nacional e internacional. Com destaque para o CBIE e RENOTE que concentram contribuições relevantes acerca de pesquisas relacionadas a Informática na Educação, que é o recorte temático deste estudo. As questões de pesquisa elaboradas são apresentadas na Tabela 1:

Tabela 1 - Questões de pesquisa.

\begin{tabular}{ll}
\hline Questões & Descrição \\
\hline QP1 & $\begin{array}{l}\text { Quais metodologias, técnicas e métodos estatísticos, softwares e algoritmos tem sido } \\
\text { utilizados em estudos no Moodle em atividades a distância? }\end{array}$ \\
QP2 & Quais são as variáveis analisadas nessas pesquisas? \\
QP3 & Quais softwares estatísticos de apoio são utilizados? \\
QP4 & Quais contribuições e limitações são apontadas nas pesquisas? \\
\hline
\end{tabular}

\subsection{String de Busca}

Elencadas as questões de pesquisa, conduziu-se a elaboração da string de busca para a obtenção de resultados primários do mapeamento sistemático (MP). As palavras-chave utilizadas foram: Educação à Distância, estatística e Moodle. Com base nesse conjunto de palavras, a string de busca construída foi: Moodle AND statistic AND (ead OR distance).

\subsection{Condução da pesquisa}

Primeiramente a busca gerou o total de 777 pesquisas. Mas, para a segunda etapa foi efetuada a filtragem dos últimos cinco anos (2015-2020), o que resultou num total de 250 trabalhos.

A partir da filtragem dos dados, passou-se para a leitura dos títulos, resumos e palavras-chave. Esse processo se faz necessário para realizar uma revisão inicial dos artigos (A), teses (T) e dissertações (D) relevantes para a pesquisa, bem como a exclusão dos trabalhos que não atendem o propósito das questões de pesquisa (QP). Nessa etapa, foram definidos e aplicados Critérios de Inclusão (CI) e Critérios de Exclusão (CE) para cada estudo. A Tabela 2 descreve tais critérios:

Tabela 2 - Critérios de Inclusão (Cl) e Critérios de Exclusão (CE)

\begin{tabular}{ll}
\hline Critérios de Inclusão & Critérios de Exclusão \\
\hline CI1: As publicações utilizam o Moodle como AVEA ? & CE1: não está em português, espanhol ou inglês. \\
$\begin{array}{ll}\text { CI2: Utiliza métodos estatísticos e/ou computacionais na } \\
\text { pesquisa? }\end{array}$ & CE2: Anterior a 2015. \\
CI3: Os estudos são de caráter educacional ? & CE3: não atende às questões de pesquisa. \\
\hline
\end{tabular}

Dos 777 estudos iniciais, restaram $59 \operatorname{estudos}^{5}$ (7,6\%). Os gráficos da Figura 1, ilustram a distribuição das pesquisas entre número de publicações ao ano e origem dos estudos (nacional, internacional). Os estudos serão selecionados somente se atenderem a esses três critérios de inclusão.

\footnotetext{
${ }^{1}$ cbie.ceie-br.org

${ }^{2}$ seer.ufrgs.br

${ }^{3}$ www.periodicos.capes.gov.br

${ }^{4}$ catalogodeteses.capes.gov.br

${ }^{5}$ Relação dos trabalhos selecionados disponível em:

https://docs.google.com/spreadsheets/d/1ANgsKEc_DznxB9dkOPMjSIOwdJZHMd6an5SW5YfLtbU/edit?usp=sharing
} 


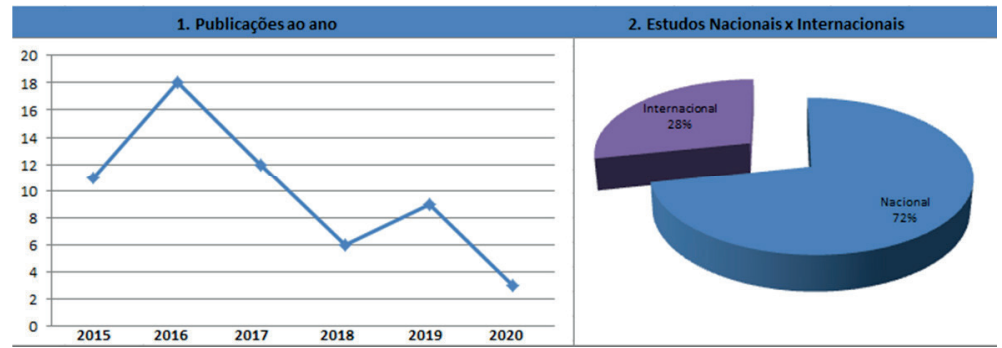

Figura 1 - Estudos selecionados por publicações ao ano e origem do estudo.

\section{Resultados e Discussões}

Como a referida pesquisa teve o interesse em investigar publicações que envolvem o uso de métodos estatísticos e computacionais em ensino a distância, utilizou-se o Moodle como ambiente de referência, pelo fato de ser um dos principais AVEA que norteiam os estudos na modalidade EaD, segundo dados obtidos no moodle.org (2020). Assim, as publicações foram classificadas e verificou-se que a grande maioria dos trabalhos realizaram suas pesquisas vinculadas ao uso da Informática na Educação (76,92\%), seguido das seguintes áreas: Educação (17\%), Saúde (3\%), Matemática (3\%) e Psicologia (2\%). As próximas seções respondem individualmente a cada questão de pesquisa.

\subsection{QP1 - Quais metodologias, técnicas, métodos estatísticos, softwares e algoritmos tem sido utilizados em estudos no Moodle, em atividades a distância?}

A QP1 tem como objetivo elencar e analisar as principais metodologias de pesquisa, técnicas e métodos estatísticos, além do uso de softwares e algoritmos computacionais presentes nos estudos selecionados. Assim, para obter dados organizados acerca dessas variáveis, foi realizada a categorização com o objetivo de classificar, tomando-se como base a proposta de Silva et al. (2015). Desse modo, a classificação é ilustrada na Tabela 3.

Tabela 3 - Métodos e ferramentas

\begin{tabular}{|c|c|c|c|c|}
\hline & & Qtd & $\%$ & Teses (T), Dissertações (D) e Artigos (A) \\
\hline \multirow{9}{*}{ Metodologia } & Descritiva & 2 & 3,39 & $\mathrm{~A} 6, \mathrm{~A} 27$. \\
\hline & Estudo de Caso & 5 & 8,47 & $\mathrm{~A} 4, \mathrm{~A} 17, \mathrm{~A} 43, \mathrm{~A} 47, \mathrm{~A} 53$ \\
\hline & Exploratória & 8 & 13,56 & $\mathrm{D} 1, \mathrm{D} 2, \mathrm{D} 3, \mathrm{~A} 3, \mathrm{~A} 6, \mathrm{~A} 10, \mathrm{~A} 28, \mathrm{~A} 36$ \\
\hline & Metaanálise & 1 & 1,69 & A23. \\
\hline & Pesquisa aplicada & 12 & 20,34 & $\mathrm{~A} 1, \mathrm{~A} 9, \mathrm{~A} 29, \mathrm{~A} 30, \mathrm{~A} 31, \mathrm{~A} 32, \mathrm{~A} 33, \mathrm{~A} 34, \mathrm{~A} 38, \mathrm{~A} 39, \mathrm{~A} 41, \mathrm{~A} 45$. \\
\hline & Pesquisa de Campo & 1 & 1,69 & A47. \\
\hline & Pesquisa Empírica & 11 & 18,64 & $\mathrm{~A} 2, \mathrm{~A} 7, \mathrm{~A} 8, \mathrm{~A} 11, \mathrm{~A} 13, \mathrm{~A} 36, \mathrm{~A} 53, \mathrm{~A} 54, \mathrm{~A} 56, \mathrm{~A} 59, \mathrm{~A} 62$. \\
\hline & Revisão Bibliográfica & 17 & 28,81 & $\mathrm{~A} 4, \mathrm{~A} 12, \mathrm{~A} 15, \mathrm{~A} 19, \mathrm{~A} 20, \mathrm{~A} 24, \mathrm{~A} 25, \mathrm{~A} 26, \mathrm{~A} 29, \mathrm{~A} 35, \mathrm{~A} 36, \mathrm{~A} 37, \mathrm{~A} 42, \mathrm{~A} 44, \mathrm{~A} 48, \mathrm{~A} 49, \mathrm{~A} 51$. \\
\hline & Revisão Sistemática & 3 & 5,08 & A35,A46,A61. \\
\hline & Análise discriminação de itens & 2 & 3,39 & D3,A7. \\
\hline & Análise Fatorial & 6 & 10,17 & $\mathrm{~A} 6, \mathrm{~A} 12, \mathrm{~A} 13, \mathrm{~A} 21, \mathrm{~A} 59, \mathrm{~A} 61$. \\
\hline & Análise Multivariada & 2 & 3,39 & $\mathrm{~A} 24, \mathrm{~A} 28$. \\
\hline & Cluster & 3 & 5,08 & $\mathrm{~A} 7, \mathrm{~A} 60, \mathrm{~A} 62$. \\
\hline & Coeficiente de correlação & 7 & 11,86 & $\mathrm{~A} 6, \mathrm{~A} 7, \mathrm{~A} 20, \mathrm{~A} 34, \mathrm{~A} 36, \mathrm{~A} 41, \mathrm{~A} 45$ \\
\hline & Estatística inferencial & 2 & 3,39 & D3,A15. \\
\hline & Medidas Descritivas & 36 & 61,02 & $\mathrm{D} 1, \mathrm{D} 2, \mathrm{D} 3, \mathrm{~T} 1, \mathrm{~A} 1, \mathrm{~A} 2, \mathrm{~A} 4, \mathrm{~A} 5, \mathrm{~A} 6, \mathrm{~A} 7, \mathrm{~A} 8, \mathrm{~A} 13, \mathrm{~A} 15, \mathrm{~A} 17, \mathrm{~A} 19, \mathrm{~A} 20, \mathrm{~A} 21, \mathrm{~A} 23, \mathrm{~A} 27, \mathrm{~A} 29$, \\
\hline Métodos e Técnicas & & & & $\mathrm{A} 31, \mathrm{~A} 34, \mathrm{~A} 35, \mathrm{~A} 37, \mathrm{~A} 38, \mathrm{~A} 43, \mathrm{~A} 46, \mathrm{~A} 47, \mathrm{~A} 48, \mathrm{~A} 49, \mathrm{~A} 50, \mathrm{~A} 51, \mathrm{~A} 52, \mathrm{~A} 53, \mathrm{~A} 54, \mathrm{~A} 55$. \\
\hline Estatísticas & PLS-SEM & 1 & 1,69 & A21. \\
\hline
\end{tabular}




\begin{tabular}{|c|c|c|c|c|}
\hline & Regressão Linear Múltipla & 6 & 10,17 & $\mathrm{~A} 3, \mathrm{~A} 11, \mathrm{~A} 12, \mathrm{~A} 13, \mathrm{~A} 20, \mathrm{~A} 26$. \\
\hline & Regressão Logística binária & 5 & 8,47 & T1,A9,A24,A42,A47. \\
\hline & SEM (equações estruturais) & 7 & 11,86 & $\mathrm{~A} 6, \mathrm{~A} 12, \mathrm{~A} 13, \mathrm{~A} 20, \mathrm{~A} 21, \mathrm{~A} 59, \mathrm{~A} 61$. \\
\hline & Série temporal & 1 & 1,69 & A30. \\
\hline & Teste não paramétricos & 1 & 1,69 & D3. \\
\hline & Teste Tukey & 1 & 1,69 & A47. \\
\hline & Teste-t & 6 & 10,17 & $\mathrm{~A} 6, \mathrm{~A} 15, \mathrm{~A} 23, \mathrm{~A} 29, \mathrm{~A} 43, \mathrm{~A} 50$. \\
\hline & Teste de Wilcoxon & 1 & 1,69 & A5 \\
\hline & Z-Score & 3 & 5,08 & A34,A36,A45. \\
\hline \multirow{7}{*}{$\begin{array}{l}\text { Recursos } \\
\text { Computacionais }\end{array}$} & Algoritmo aprendizado de máquina: & 11 & 18,64 & $\mathrm{~A} 4, \mathrm{~A} 9, \mathrm{~A} 10, \mathrm{~A} 30, \mathrm{~A} 31, \mathrm{~A} 32, \mathrm{~A} 33, \mathrm{~A} 36, \mathrm{~A} 42, \mathrm{~A} 43, \mathrm{~A} 55$. \\
\hline & Algoritmo de Clusterização: & 3 & 5,08 & $\mathrm{~A} 25, \mathrm{~A} 36, \mathrm{~A} 60$ \\
\hline & Algoritmos de Classificação: & 3 & 5,08 & A9,A30,A42. \\
\hline & Desenvolvimento aplicações Web: & 2 & 3,39 & A39,A49. \\
\hline & Linguagem Programação: & 7 & 11,86 & A1,A25,A39,A42,A45,A49,A55. \\
\hline & Pacote R Studio: & 1 & 1,69 & A38. \\
\hline & Plugin moodle: & 2 & 3,39 & A38,A39. \\
\hline
\end{tabular}

A partir da visualização dos dados da Tabela 3, observa-se que a maioria dos trabalhos utilizaram as metodologias de pesquisa: revisão bibliográfica $(28,81 \%)$, como forma de fundamentar teoricamente os estudos realizados. Em números menores aparecem as metodologias de pesquisa aplicada $(20,34 \%)$ e pesquisa exploratória $(13,56 \%)$.

Em relação aos métodos e técnicas estatísticas, destacam-se os estudos que utilizam Medidas Descritivas (61,02\%), seguido pelo uso de Equações Estruturais (11,86\%), Coeficiente de Correlação $(11,86 \%)$, Análise Fatorial e Regressão Linear Múltipla (10,17\%), dentre outras técnicas registradas com proporções menores.

O uso de Medidas Descritivas nos trabalhos envolvendo atividades educacionais no Moodle, merecem destaque, pois além do registro de uso em larga escala nas pesquisas analisadas, o uso dessa técnica, permite que o investigador realize um resumo, descrevendo e compreendendo os dados das amostras realizadas nas pesquisas. Ao fazer uso de medidas de tendência central (média, mediana e moda), medidas de dispersão (valores mínimo e máximo, desvio padrão e variância) e medidas de distribuição (achatamento e simetria da curva), facilita o processo de análise e permite que essa informação se torne manejável, facilitando comparações e relações entre os elementos da pesquisa.

Nota-se que algumas pesquisas aparecem em mais de uma categoria, pois o uso de técnicas estatísticas estão normalmente agregadas a uma metodologia de pesquisa e utilizam também vários métodos estatísticos. Especialmente em pesquisas com um enfoque quali-quantitativo, onde as técnicas estatísticas vem com o objetivo de complementar as análises e reflexões no formato de números e gráficos.

No que tange a utilização de recursos computacionais, destacam-se algumas linguagens de programação $^{6}(11,86 \%)$, onde sua utilização está justificada por auxiliar e potencializar o processo de análise estatística e implementar alternativas automatizadas de relações e comparações entre as variáveis dos estudos.

Em relação aos algoritmos utilizados, destacam-se os Algoritmos de Aprendizado de Máquina $(18,64 \%)$. Em proporções menores registram-se o uso de algoritmos de clusterização e Classificação

\footnotetext{
${ }^{66}$ Dentre as linguagens de programação utilizadas nas pesquisas, registra-se o uso da plataforma Java, Javascript, PHP, SQL e Python.
} 
(5,08\%), aplicativos de desenvolvimento Web (3,39\%), desenvolvimento de Plugins para o Moodle $(3,39 \%)$ e pacotes utilizados no $R$ Studio $(1,69 \%)$.

\subsection{QP2 - Quais variáveis são analisadas nessas pesquisas?}

Respondendo a segunda questão registrada na QP2, procurou-se listar todas as variáveis ${ }^{7}$ presentes nas pesquisas que envolvem o uso de métodos estatísticos e computacionais utilizando o Moodle. Pelo fato da pesquisa utilizar um ambiente virtual no processo de ensino e aprendizagem, a grande maioria registrou estudos relacionados a variável de Interação (57,63\%), Aprendizagem (47,46\%) e Interatividade $(35,59 \%)$ conforme registros da Tabela 4.

\section{Tabela 4 - Variáveis de estudo}

\begin{tabular}{|c|c|c|c|}
\hline Variáveis & Qtd & $\%$ & Teses (T), Dissertações (D) e Artigos (A) \\
\hline Aprendizagem & 28 & 47,46 & $\begin{array}{l}\text { D1,D2,D3,T1,A1,A4,A6,A8,A10,A15,A17,A19,A20,A21,A23, } \\
\text { A24,A28,A29,A36,A39,A43,A3,A7,A9,A49,A54,A60,A61. }\end{array}$ \\
\hline Aspectos Cognitivos & 3 & 5,08 & $\mathrm{~A} 7, \mathrm{~A} 8, \mathrm{~A} 30$ \\
\hline Aspectos Culturais & 1 & 1,69 & A13 \\
\hline Autodeterminação & 4 & 6,78 & A6,A12,A30,A59 \\
\hline Autoregulação & 5 & 8,47 & $\mathrm{~A} 10, \mathrm{~A} 29, \mathrm{~A} 30, \mathrm{~A} 59, \mathrm{~A} 61$ \\
\hline Avaliação & 7 & 11,86 & $\mathrm{D} 2, \mathrm{D} 3, \mathrm{~A} 13, \mathrm{~A} 27, \mathrm{~A} 28, \mathrm{~A} 43, \mathrm{~A} 53$ \\
\hline Colaboração & 11 & 18,64 & D1,A6,A7,A11,A12,A20,A23,A26,A35,A39,A47 \\
\hline Comportamento estudante & 4 & 6,78 & $\mathrm{~A} 7, \mathrm{~A} 51, \mathrm{~A} 59, \mathrm{~A} 60$ \\
\hline Comunicação & 8 & 13,56 & D1,A11,A12,A26,A29,A47,A51,A52 \\
\hline Desempenho Estudante & 17 & 28,81 & D1,D2,D3,T1,A20,A21,A23,A24,A25,A27,A30,A41,A42,A3,A4,A9,A10 \\
\hline Distância transacional & 1 & 1,69 & A47 \\
\hline Engajamento estudante & 6 & 10,17 & $\mathrm{D} 1, \mathrm{~T} 1, \mathrm{~A} 9, \mathrm{~A} 21, \mathrm{~A} 47, \mathrm{~A} 49$ \\
\hline Engajamento Professor & 6 & 10,17 & $\mathrm{D} 1, \mathrm{~A} 8, \mathrm{~A} 12, \mathrm{~A} 29, \mathrm{~A} 49, \mathrm{~A} 56$ \\
\hline Engajamento tutor & 10 & 16,95 & D1,A12,A25,A29,A34,A36,A43,A45,A49,A54 \\
\hline Evasão & 7 & 11,86 & T1,A3,A24,A25,A30,A42,A44 \\
\hline Fluência Digital & 6 & 10,17 & $\mathrm{~A} 2, \mathrm{~A} 13, \mathrm{~A} 21, \mathrm{~A} 51, \mathrm{~A} 56, \mathrm{~A} 60$ \\
\hline Formação Professores & 2 & 3,39 & D2,A37 \\
\hline Gênero & 1 & 1,69 & A13 \\
\hline Hábitos Estudo & 3 & 5,08 & $\mathrm{~A} 6, \mathrm{D} 2, \mathrm{~T} 1$ \\
\hline Idade & 1 & 1,69 & D2 \\
\hline Infraestrutura & 1 & 1,69 & A17 \\
\hline \multirow[t]{2}{*}{ Interação $^{8}$} & 34 & 57,63 & $\mathrm{D} 1, \mathrm{~T} 1, \mathrm{~A} 2, \mathrm{~A} 5, \mathrm{~A} 6, \mathrm{~A} 7, \mathrm{~A} 10, \mathrm{~A} 11, \mathrm{~A} 12, \mathrm{~A} 15, \mathrm{~A} 19, \mathrm{~A} 20, \mathrm{~A} 23, \mathrm{~A} 24, \mathrm{~A} 26, \mathrm{~A} 27$, \\
\hline & & & A28,A29,A30,A32,A33,A34,A35,A36,A38,A39,A41,A43,A44,A45,A47,A50,A52,A62. \\
\hline Interatividade ${ }^{9}$ & 21 & 35,59 & D1,A2,A6,A7,A10,A12,A15,A19,A24,A29,A30,A32,A33,A34,A37,A38,A45,A47,A48,A50,A52 \\
\hline Mediação & 6 & 10,17 & $\mathrm{D} 2, \mathrm{~T} 1, \mathrm{~A} 10, \mathrm{~A} 12, \mathrm{~A} 21, \mathrm{~A} 27$ \\
\hline Motivação & 2 & 3,39 & A6,A7 \\
\hline Perfil Estudante & 8 & 13,56 & $\mathrm{D} 2, \mathrm{~T} 1, \mathrm{~A} 31, \mathrm{~A} 6, \mathrm{~A} 13, \mathrm{~A} 50, \mathrm{~A} 51, \mathrm{~A} 60$ \\
\hline Plágio & 1 & 1,69 & A46 \\
\hline Planejamento tempo & 1 & 1,69 & A8 \\
\hline Problematização & 4 & 6,78 & $\mathrm{D} 1, \mathrm{~A} 11, \mathrm{~A} 20, \mathrm{~A} 51$ \\
\hline
\end{tabular}

\footnotetext{
${ }^{7}$ Elementos e temas centrais das pesquisas realizadas.

${ }^{8}$ Interação: ações que exigem intersubjetividade, relação sujeito-sujeito, Ex. participação de fóruns, chats, videoconferência, wiki.

${ }^{9}$ Interatividade: ações de relação entre sujeito e tecnologia. Ex. acesso a arquivos.
} 


\begin{tabular}{lrrl} 
& CINTED-UFRGS & Revista Novas Tecnologias na Educação \\
\cline { 2 - 4 } & 1 & 1,69 & A5 \\
Satisfação & 2 & 3,39 & A23,A29 \\
Suporte técnico & 6 & 10,17 & A5,A15,A17,A19,A37,A48 \\
Usabilidade & 7 & 11,86 & A1,A8,A29,A31,A32,A33,A47 \\
Uso das tecnologias & 7 &
\end{tabular}

Outras variáveis também merecem um destaque como o desempenho do estudante $(28,81 \%)$, atividades que envolvem a Colaboração $(18,64 \%)$ e engajamento do tutor $(16,95 \%)$. São também elementos relevantes, que envolvem o acompanhamento do tutor nas atividades a distância e a importância das atividades colaborativas no Moodle.

\subsection{QP3 - Quais são os softwares estatísticos utilizados nas análises das pesquisas?}

Dentre os estudos analisados, destaca-se que as pesquisas muitas vezes utilizam mais de um software estatístico na resolução de problemas. Isso foi justificado pelo fato de todas trabalharem com várias técnicas (medidas descritivas, análise fatorial, covariância, regressões). O software Excel, contando com todas suas versões, corresponde o total de $(33,90 \%)$ de utilização das pesquisas realizadas. Seguido pelo R $(28,81 \%)$ e dos aplicativos utilizados também em cálculos estatísticos como o SPSS (22,03\%), LibreOffice Calc (15,25\%), SPSS AMOS e Stata (3,39\%) e SmartPLS e MPlus 7 (1,69\%), conforme registros da Tabela 5 .

Tabela 5 - Softwares estatísticos utilizados

\begin{tabular}{llll}
\hline Softwares & Qtd & \multicolumn{1}{c}{$\%$} & Teses (T), Dissertações (D) e Artigos (A) \\
\hline Excel & 20 & 33,90 & $\mathrm{D} 1, \mathrm{D} 2, \mathrm{~A} 1, \mathrm{~A} 6, \mathrm{~A} 8, \mathrm{~A} 27, \mathrm{~A} 29, \mathrm{~A} 30, \mathrm{~A} 31, \mathrm{~A} 33, \mathrm{~A} 34, \mathrm{~A} 35, \mathrm{~A} 36, \mathrm{~A} 39, \mathrm{~A} 43, \mathrm{~A} 46, \mathrm{~A} 48, \mathrm{~A} 51, \mathrm{~A} 52, \mathrm{~A} 54$. \\
\hline LibreOffice Calc & 9 & 15,25 & $\mathrm{~T} 1, \mathrm{~A} 25, \mathrm{~A} 32, \mathrm{~A} 37, \mathrm{~A} 45, \mathrm{~A} 49, \mathrm{~A} 53, \mathrm{~A} 55, \mathrm{~A} 56$. \\
\hline MPlus 7 & 1 & 1,69 & $\mathrm{~A} 13$. \\
\hline R & 17 & 28,81 & $\mathrm{~A} 2, \mathrm{~A} 3, \mathrm{~A} 4, \mathrm{~A} 5, \mathrm{~A} 7, \mathrm{~A} 9, \mathrm{~A} 10, \mathrm{~A} 11, \mathrm{~A} 38, \mathrm{~A} 41, \mathrm{~A} 42, \mathrm{~A} 44, \mathrm{~A} 47, \mathrm{~A} 50, \mathrm{~A} 59, \mathrm{~A} 60, \mathrm{~A} 61$. \\
SmartPLS & 1 & 1,69 & $\mathrm{~A} 21$. \\
\hline SPSS & 13 & 22,03 & $\mathrm{D} 3, \mathrm{~A} 6, \mathrm{~A} 12, \mathrm{~A} 15, \mathrm{~A} 17, \mathrm{~A} 20, \mathrm{~A} 21, \mathrm{~A} 23, \mathrm{~A} 24, \mathrm{~A} 26, \mathrm{~A} 28, \mathrm{~A} 34, \mathrm{~A} 62$. \\
SPSS AMOS & 2 & 3,39 & $\mathrm{~A} 12, \mathrm{~A} 20$. \\
\hline Stata & 2 & 3,39 & $\mathrm{~T} 1, \mathrm{~A} 24$. \\
\hline
\end{tabular}

Importante registrar que o software SPSS foi mais utilizado em cálculos envolvendo medidas descritivas e regressões lineares e o SPSS AMOS na elaboração de modelos estruturais envolvendo relações entre as variáveis de estudo.

\subsection{QP4 - Quais contribuições e limitações são apontadas nas pesquisas?}

Dos resultados obtidos, a maioria das contribuições das pesquisas estão direcionadas em ações envolvendo o acompanhamento de estudantes $(59,32 \%)$, aplicações de inovação tecnológica $(25,42 \%)$ e o aprendizado online (20,34\%). Ilustradas com maiores detalhes na Tabela 6.

Tabela 6 - Contribuições e limitações das pesquisas

\begin{tabular}{lccll}
\hline Contribuições / Limitações & Qtd & $\%$ & Teses (T), Dissertações (D) e Artigos (A) \\
\hline Contribuições & & & \\
Acompanhamento Estudantes & 35 & 59,32 & T1,A2,A3,A4,A6,A7,A9,A10,A11,A12,A13,A17,A24,A25,A26,A28,A30,A31, \\
& & & A32,A33,A34,A35,A36,A37,A38,A41,A42,A44,A45,A46,A47,A51,A59,A61,A62. \\
Aprendizado online & 12 & 20,34 & D1,D2,D3,A10,A20,A23,A25,A36,A39,A50,A60,A61 \\
Avaliação & 6 & 10,17 & D2,D3,A13,A27,A28,A53 \\
Colaboração & 10 & 16,95 & D1,A6,A7,A11,A20,A23,A35,A39,A47,A51 \\
Comunicação & 6 & 10,17 & D1,D2,A11,A19,A33,A51 \\
Desempenho estudantes & 6 & 10,17 & T1,A21,A24,A25,A41,A3
\end{tabular}




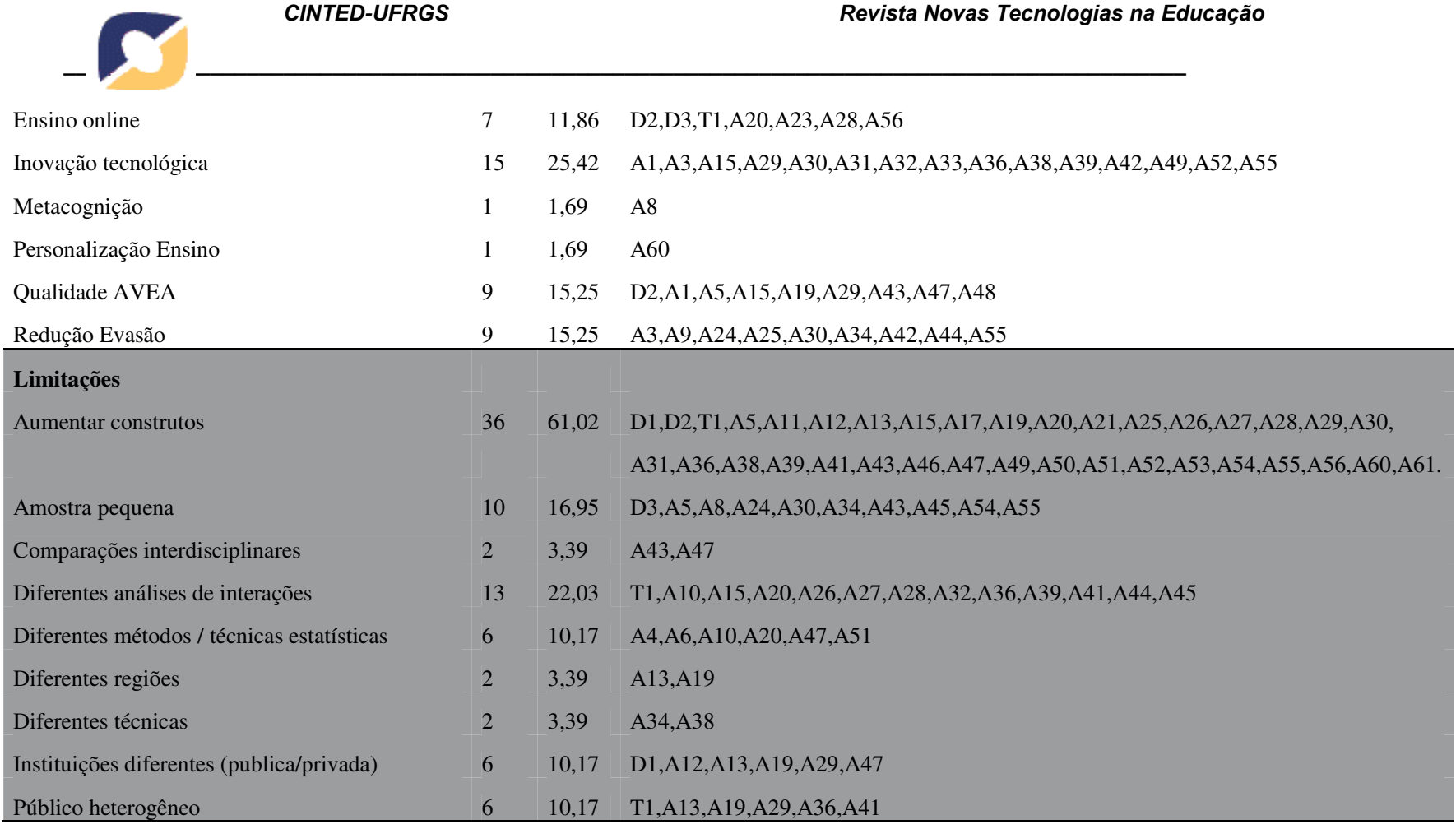

A partir desse detalhamento, observaram-se também elementos importantes que possuem relação com atividades educacionais no Moodle. Contribuições referentes ao processo colaborativo dos estudantes, foi relatada em $16,95 \%$ dos estudos.

Conforme estudos de Schmitt et al.(2019)[A55], um dos maiores obstáculos enfrentados na modalidade $\mathrm{EaD}$ é a evasão dos estudantes. Esses números podem chegar na faixa de até $25 \%$ (Associação Brasileira de Educação a Distância, 2014), sendo que um dos principais fatores que aumentam esse índice é a falta de acompanhamento dos estudantes.

Dentre as contribuições observadas nas pesquisas, o maior índice foi registrado no ítem Acompanhamento dos Estudantes, atingindo cerca de 59\% dos estudos, uma vez que o Moodle tende a registrar um volume grande de informações dos estudantes (acessos, visualizações, participações em atividades, notas e demais ações). Assim, seu banco de dados torna-se um repositório importante para a tomada de decisões da equipe gestora, professores e tutores. Com o uso de técnicas, métodos estatísticos e recursos computacionais, esses dados permitem propor inovações tecnológicas([A1],[A3],[A15],[A29],[A30],[A31],[A32],[A33],[A36],[A38],[A39],[A42],[A49],[A52] ,[A55]), ao qual permitem trabalhar com previsões de desempenho, evasão e identificação de problemas de aprendizagem online ${ }^{10}$ (Cooper,2012).

Contribuições referentes ao processo de Colaboração é destacado nos estudos ([D1],[A6],[A7],[A11],[A20],[A23],[A35],[A39],[A47],[A51]), atingindo 16,95\% dos trabalhos durante o MS. Segundo Cunha e Siebra (2015)[A35], estudos destacam a necessidade de que os estudantes partam do aprendizado individual para o trabalho em grupo. Interagir de maneira colaborativa para solucionar problemas pode ter consideráveis vantagens. Primeiro, através do ensino recíproco (Rosenshine e Meister, 1994), a troca de conhecimento é estabelecida mais fortemente; estudantes mais experientes podem apoiar aos demais. Assim, acredita-se no trabalho em grupo para, de fato, elevar a aprendizagem e potencializar a qualidade do processo educacional na EaD.

Algumas limitações relacionadas merecem destaque, uma que $61,02 \%$ das pesquisas relatam a possibilidade de estudos com um número maior de construtos (variáveis), aumentando assim o alcance

\footnotetext{
${ }^{10}$ Dentre os estudos realizados no MS, as contribuições referentes a Aprendizagem online atingiram 20,34\% das pesquisas ([D1],[D2],[D3],[A10],[A20],[A23],[A25],[A36],[A39],[A50],[A60],[A61]).
} 
das contribuições dessas variáveis em relação ao processo de ensino e aprendizagem na EaD. Na sequência, aparece a possibilidade de trabalhos futuros realizarem pesquisas contendo diferentes análises nas interações do Moodle (22,03\%). Assim, registrando a necessidade de usufruir da variedade de informação disponível no banco de dados das ações dos sujeitos da $\mathrm{EaD}$ (professor, tutor e estudante) que o Moodle oferece, propondo estudos que tragam informações relevantes e que possam auxiliar no processo de acompanhamento do estudante, diminuição da evasão e aperfeiçoamento de ações vinculadas ao processo de ensino e aprendizagem.

\section{Considerações Finais}

Esse estudo apresentou um Mapeamento Sistemático da Literatura envolvendo metodologias, técnicas e softwares estatísticos, recursos computacionais, contribuições e limitações no campo teórico, prático e variáveis relacionadas com a utilização de métodos estatísticos e computacionais em pesquisas educacionais no Moodle, entre os anos de 2015 e 2020. De um total de 777 trabalhos préselecionados, 59 foram tabulados e avaliados, centrando-se naqueles que abordaram os objetivos do estudo.

As publicações englobaram várias áreas do conhecimento, registrando uma ampla maioria vinculada em estudos educacionais com ênfase na área de informática na educação.

Diante os resultados obtidos ao longo da pesquisa, conclui-se que, a maioria dos estudos são provenientes de pesquisas realizadas no Brasil e estão distribuídos nos principais eventos, portais e periódicos utilizados pela comunidade científica na área de tecnologias educacionais.

Do ponto de vista do problema destacado, a literatura analisada durante o MS apresentou várias pesquisas que extraem informações referentes a recursos e atividades do Moodle, como:

Avaliação de atividades de tutores [A25], análise exploratória de atividades que mais contribuem para a construção colaborativa do conhecimento [A26], extração no banco de dados do Moodle para identificar estudantes com potencial de evasão [A30], arquitetura multiagente para acompanhamento de atividades baseada em Learning Analytics (LA) [A36], integração do Moodle com o framework Shiny para desenvolvimento de LA [A38], uso do plugin Glossário LV para avaliação usando conceitos de Learning Vectors (LV) [A39], predição precoce de evasão utilizando mineração de dados relativos as interações dos estudantes [A44], entre outros.

No que tange o ponto culminante da pesquisa, relacionada ao uso de métodos estatísticos e recursos computacionais em atividades educacionais no Moodle, destacam-se alguns: na estatística, o uso das medidas descritivas e na área computacional, o uso de algoritmos de aprendizado de máquina e linguagens de programação para automatizar ações no Moodle. Outros recursos computacionais como o uso de plug-ins no Moodle, uso de pacotes personalizados do $R$ studio e aplicativos de desenvolvimento Web, também proporcionaram criar inovações tecnológicas diante o processo de ensino e aprendizagem.

Dentre os recursos e algoritmos observados na pesquisa, alguns merecem destaque: Algoritmo K-means ([A25],[A36],[A60]), utilizado em avaliações comportamentais de estudantes dentro do Moodle realizando classificações por meio de agrupamento de dados. A escolha deste algoritmo se deu pelo bom desempenho, tempo de execução e simplicidade na interpretação dos resultados. Weka ([A4],[A10],[A30],[A33],[A36],[A43],[A55]), ferramenta que disponibiliza uma coleção de algoritmos de aprendizado de máquina, escrita na plataforma Java, para utilização na mineração de dados. Nele estão implementadas as técnicas utilizadas nas pesquisas, assim como outras técnicas de aprendizagem de máquina. 
Com esses recursos computacionais, se torna possível que coordenadores de cursos, professores e tutores possam refletir sobre os desempenhos obtidos. Além disso, esse recurso facilita o monitoramento dos estudantes em tempo real, de modo que estratégias pedagógicas possam ser (re)planejadas para aperfeiçoar o ensino, propiciar a participação ativa e diminuir evasões e reprovações.

Dentre as variáveis utilizadas nos estudos, destacaram-se: aprendizagem, desempenho de estudantes e recursos de interação e interatividade.

Dessa forma, conclui-se que, várias foram as contribuições das pesquisas, ressaltando a questões envolvendo: o acompanhamento dos estudantes no ambiente (facilitado com o uso de recursos estatísticos e algoritmos) e as inovações tecnológicas elaboradas nas pesquisas, implementadas através do uso de linguagens de programação e aplicações de desenvolvimento Web.

\section{Referências}

ASSOCIAÇÃO BRASILEIRA DE EDUCAÇÃO A DISTÂNCIA (ABED). Disponível em < http://www.abed.org.br/censoead2014/CensoEAD2014_portugues.pdf> Acesso em: 15 de Junho de 2020.

COOPER, A. What is analytics? Definition and essential characteristics. CETIS Analytics Series, $1(5), 1-10,2012$.

CUNHA, F. CLAUIRTON SIEBRA. Especificação de Perfis de Grupos para Suporte à Formação de Grupos Colaborativos na EAD. Congresso Brasileiro de Informática na Educação - CBIE, 2015.

KITCHENHAM, B. AND CHARTERS, S.: Guidelines for performing systematic literature reviews in software engineering. Technical Report EBSE 2007-001, Keele University and Durham University Joint Report, 2007.

RICHTER, CLEITOM J. O Ensino de Programação mediado por Tecnologias Educacionais: uma Revisão Sistemática de Literatura. Revista novas tecnologias na Educação - CINTED-UFRGS, 2019.

ROMERO, C., VENTURA, S. E GARCÍA, E. Data mining in course management systems: Moodle case study and tutorial. Computers and Education, 51 (1), pp. 368-384, 2008.

ROSENSHINE B., MEISTER, C. Reciprocal Teaching: A Review of the Research, Rev. of Educational Research, vol. 64, pp. 479-530, 1994.

SCHMITT J. A., BERNARDI G., KANTORSKI G. Z., iPermanency: uma ferramenta para gestão da evasão na EaD baseada em Mineração de Dados Educacionais, Revista novas tecnologias na Educação - CINTED-UFRGS, 2019.

SOUZA, R. , MENDES, F. N., SANTOS, A., LAYSA FONTES, EFRAIM NAASSOM, VALENTIM, R. Um Ambiente Inteligente de Avaliação de Comporta de Tutores e Turmas no Ambiente Virtual de Aprendizagem Moodle. Congresso Brasileiro de Informática na Educação CBIE, 2016.

WEISS, S. M. E KULIKOWSKI, C. A. Computer Systems that Learn: Classification and Prediction Methods from Statistics, Neural Nets, Machine Learning, and Expert Systems. Morgan Kaufmann Publishers Inc., São Francisco, CA, USA, 1991. 